\title{
Video Error Concealment Based on Data Hiding for the Emerging Video Technologies
}

\author{
Francisco Aguirre-Ramos, Claudia Feregrino-Uribe, and Rene Cumplido \\ Coordinación de Ciencias Computacionales, \\ Instituto Nacional de Astrofísica, Óptica y Electrónica. \\ Luis Enrique Erro no. 1, 72840 Tonantzintla, Puebla, Mexico \\ \{fjaguirre, cferegrino, rcumplido\}@inaoep.mx \\ http://www.inaoep.mx
}

\begin{abstract}
The new high efficiency video coding standard (HEVC) includes structures and tools that were not available in previous standards. The macrobock concept was replaced by a quad-tree structure that includes: coding units, prediction units and transform units; also, new parallelization tools are now available. Video transmissions over error prone environments have the need of reliable and efficient error concealment methods. Unfortunately, most of the existent error concealment methods interfere, or do not take advantage of the new structures and tools.

In this work, a data hiding based error concealment method is proposed for the HEVC. During encoding, information is embedded into the residual transform coefficients; this information, is later retrieved and used during the error concealment process. The performed experiments and results show a superior performance when compared against the non-normative error concealment method included in the H.264/AVC joint model.
\end{abstract}

Keywords: Error concealment, Data hiding, HEVC, Quad-tree, H.264.

\section{Introduction}

Video technologies constantly evolve, new applications emerge everyday and the video compression technologies have to be capable of following the same evolution rhythm to provide compression solutions to the new video applications. Recently, in a combined effort, the ITU-T Video Coding Experts Group and the ISO/IEC Moving Picture Experts Group, created the Joint Collaborative Team on Video Coding (JCT-VC) with the aim of developing the new video coding standard High Efficiency Video Coding (HEVC) [5].

It is predicted that in some years, HEVC will replace H.264/AVC [12] in most video applications. HEVC introduces some new characteristics such as the quadtree structure and parallel encoding/decoding tools. Also, some characteristics that were available in previous standards are taken away; one of them, flexible macroblock ordering (FMO), was the basis of many error concealment (EC) methods [15, 19]. 
EC methods are designed to deal with lost parts of video that are the product of a failure in the storage media or packet losses during transmission of the coded bit-string. These methods can recover inter coded frames, intra coded frames, or both; in previous standards, intra frames were notably more important than inter frames. In HEVC the inter frames have an increased importance due to the redesign made to the open group of pictures (GOP) and random access features.

There are many approaches for inter EC (also named temporal EC), some of them [14, 13, 19, 17, 15, 18] create a set of candidate motion vectors (MVs) to replace the lost block and select the best candidate by using some distortion measure, these candidates can belong to neighboring blocks [14, 17] or be artificially created $[13,19,15,18$. Some other authors have proposed to perform the concealment using a reduced block size [20, 17, 16], instead of the basic block size $(16 \times 16$ in H.264/AVC and $64 \times 64$ in HEVC) they use smaller partitions to achieve better approximations in areas with complex motion or high detail.

The previously mentioned methods had an acceptable performance when working in H.264/AVC but, to achieve their results, they mostly rely on two features that are no longer available in HEVC. First, the block size, in H.264/AVC and previous standards, had been $16 \times 16$ samples; now, in HEVC the size was increased to $64 \times 64$ and linked to a complex quad-tree structure. The other feature that is not longer available in HEVC is the FMO, this tool was useful to increase the resiliency of slices when transmitted over error prone environments and allowed the possibility of correctly receiving the top, left, bottom and right block neighbors. Also, new structures were introduced to allow parallel encoding/decoding; this interferes with the slice interleaving schemes used in most of the EC methods in the literature.

In this work, an inter error concealment method, based on reversible data embedding is proposed. Information is embedded in the video during the encoding process, this information is retrieved and used in the decoding stage to conceal lost parts of video; the proposal takes into account the newly introduced features in HEVC such as quad-tree partitioning, coding units, prediction units, transform units, and parallel structures. Experimental results show an increase over the widely known inter error concealment method present in the H.264/AVC joint model.

The paper is organized as follows; in section 2, the embedded information and the details of the reversible embedding scheme are given. In section 3 , the information retrieval process and the concealment process is presented. The experiments, results, and comparisons are included in section 4, Finally, in section 5. conclusions of the present work are presented.

\subsection{The HEVC Standard}

The Joint Collaborative Team on Video Coding (JCT-VC) is in charge of the creation and standardization of the new HEVC, which in January 2013 was in the first stage of approval [3]. HEVC, named H.265 by the ITU-T and MPEG-H part 2 by the ISO/IEC, is designed to provide up to $50 \%$ of increased coding efficiency when compared to the H.264/AVC video coding standard. 
The compression efficiency gain in HEVC is the result of many optimizations that report small gains in the coding process. Also, HEVC encoder has an increased complexity of about 10 times when compared with H.264/AVC [4].

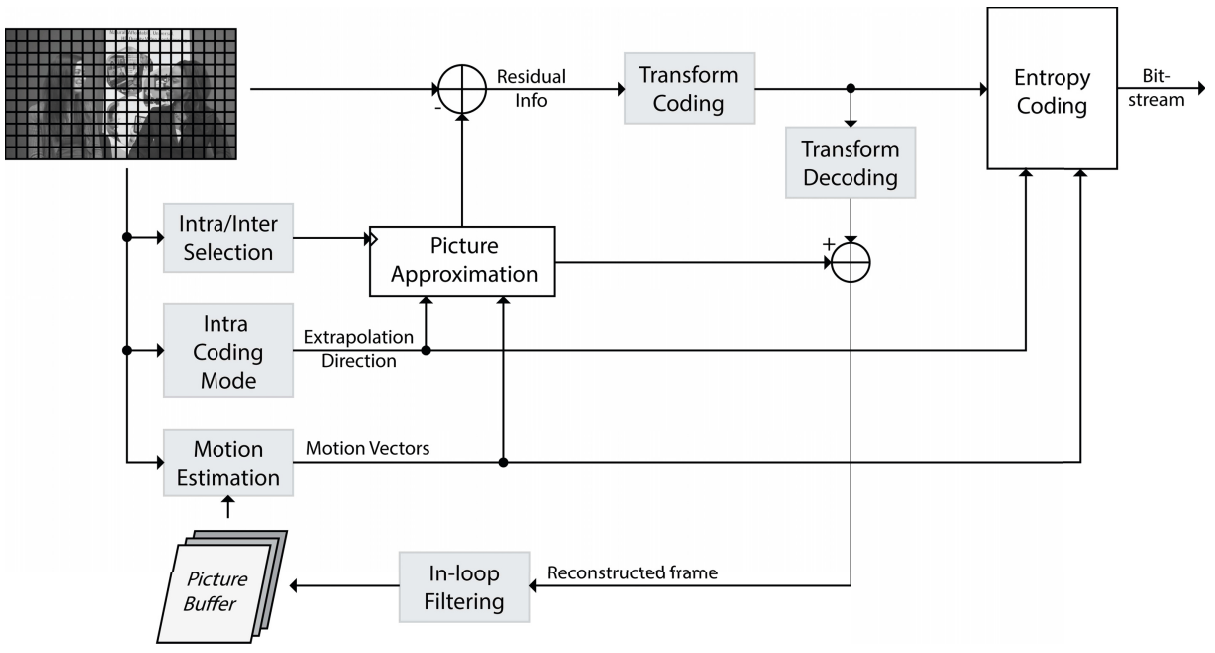

Fig. 1. Basic HEVC encoder block diagram

A basic diagram of the HEVC encoding process is shown in figure 1, broadly explained, the process includes:

- Partitioning. As a first step, each frame is partitioned into the basic block unit, now named coding unit (CU); coding units can be as small (SCU) as $8 \times 8$ or as large (LCU) as $64 \times 64$ samples, depending on the encoding configurations. In previous standards, the basic block unit was the macroblock $(\mathrm{MB})$ with a maximum size of $16 \times 16$ pixels. Also, each LCU can be recursively partitioned into smaller CUs; this process generates tree-like structures named quad-tree structures. The quad-tree structure is one of the most relevant characteristics of HEVC and helps to provide better approximations to the object shapes in frame.

- Intra/Inter coding. A block level decision of the coding mode is carried out based on distortion measures, the idea is to achieve the minimum distortion (at LCU-level) while keeping the bit rate within a defined constrained level. Once the coding mode has been defined, the intra or inter coding information is calculated.

- Intra information. For intra coding, similar to H.264/AVC, an intra coding mode is calculated; many modes are calculated per LCU and there are 35 different intra modes to chose from (for luma) [5]. Each CU has a prediction unit $(\mathrm{PU})$ shape assigned. PUs are another sub-partitioning structure introduced in HEVC, they help to approximate more closely 
the shape of objects in the image. There are different PUs available for each coding mode (intra and inter) and each of them has its own coding restrictions [4].

- Inter information. Inter coding requires of the calculation of motionvectors (MVs), the motion-estimation process is carried out at PU partition level. There is a higher number of PU shapes available for inter coding than for intra coding; up to two MVs are calculated per PU partition, increasing the accuracy of the motion-estimation process and reducing significantly the residuals.

Inter-prediction

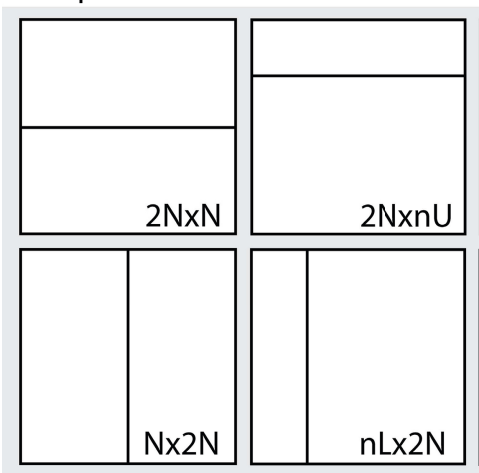

Intra-prediction

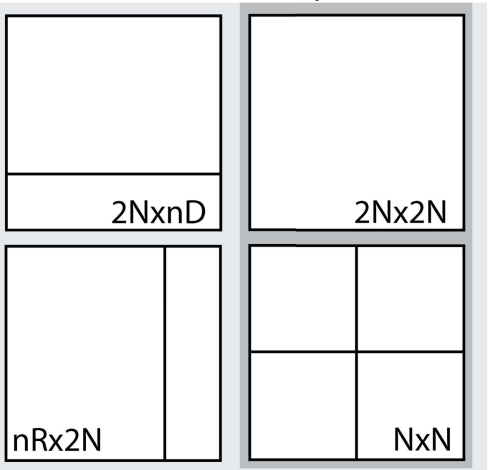

Fig. 2. Allowed PUs shapes in HEVC, all the shown PUs are available for inter coding but only the last two $(2 N \times 2 N$ and $N \times N)$ are alowed for intra coding

In figure 2, the available PUs shapes for each of the coding modes are shown. Notice that some asymmetrical PUs were introduced for inter coding, allowing better approximations in the motion-estimation process than in previous standards.

- Residual calculation. The residuals are calculated between the original block and its approximation at CU level. For some inter coding modes, residuals are not coded.

- Transform coding. The residual block is transform-coded, this is achieved by sub-partitioning each CU residual block into transform units (TU). The partitioning scheme can be recursive, similar to the $\mathrm{CU}$ partitioning. There are different sizes of TUs, they can be as large as $32 \times 32$ or as small as $4 \times 4$, this allows an increased efficiency in residual coding.

- Entropy coding. All the required reconstruction information is coded using context-adaptive binary arithmetic coding (CABAC) in any of its operating modes: regular and bypass [6].

- Reconstruction and buffering. The transmitted picture is reconstructed at encoder. This includes all in-loop filtering process, the resulting picture is a reproduction of the one reconstructed at decoder's side. The reproduced 
frame is buffered and used later as a reference picture during the inter coding process.

Many different features were added to HEVC, some of them aim at creating error resilient bit-strings; allowing the decoding of damaged sequences and avoiding error propagation at bit-string level. Nonetheless, sequences decoded from damaged bit-strings keep having the same issues related to error propagation due to inter and intra coding. In fact, the problem is worst because of the increased block size in HEVC; most of the state of the art error concealment techniques do not perform well with the new size.

\section{Proposed Method: Encoder}

The operations performed by the proposed data hiding error concealment (DHEC) method can be divided according to where they are carried out: encoding or decoding stage. In the encoder, the information that will be used to conceal the lost parts in each frame during decoding, is embedded into the video using the proposed multi-level embedding scheme.

The information is embedded exclusively into the DCT coefficients of the residual blocks, the embedding technique is fully reversible, so the original media can be recovered at decoder if the sequence was successfully transmitted without errors.

\subsection{Information to Embed}

During the design of a DHEC method, many important decisions are taken, maybe the most important, is which information will be embedded into the media. This information has to be carefully selected to be lightweight but useful during the error concealment stage. An excess in the amount of embedded information will cause a considerable increment in the video bit rate; on the other hand, if the information is lightweight but not useful enough, its embedding will only represent a disadvantage.

The proposed method embeds the complete LCU partitioning structure, including the PU partitions. This information is available during the encoding stage and can be used to improve the reconstruction during concealment of damaged areas in frame.

The coding scheme is simple, each partitioning level in the LCU structure is coded using ' 1 '; when the maximum depth in the partition branch has been reached, ' 0 ' is coded. After each ' 0 ', the corresponding PU structure is coded using the same variable-length codes that are used during the encoding stage in the Test Model under Consideration (TMuC) of HEVC [7], with a maximum requirement of 5 bits per PU shape. In figure 3, a graphical example of a LCU partitioning structure and its associated Tree-CU (TCU) is shown, the bit-string that represents the LCU structure is: 1110000100000011000000100001000010000 . In the example bit-string, the PU codes are missing; as it was aforementioned, PU codes should be located after each ' 0 ' in the bit-string. 


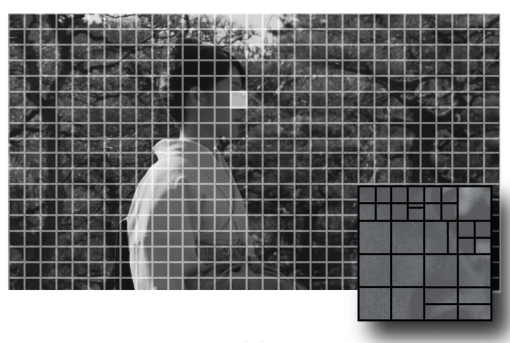

(a)

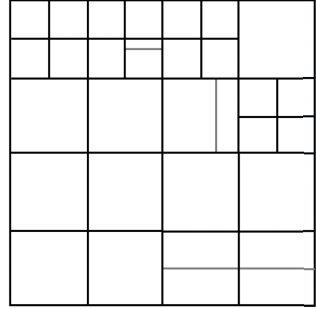

(b)

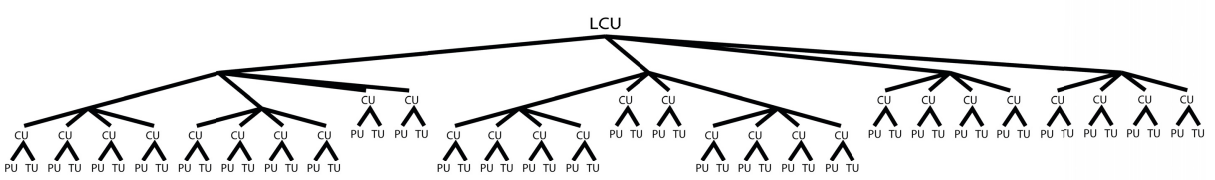

(c)

Fig. 3. Frame partitioning example in HEVC. (a) Frame partitioned into $64 \times 64$ LCUs, at the same time each LCU has its own structure. (b) Example of a LCU partitioning structure. (c) TCU of the structure shown in (b).

In the worst case, a maximum of 85 bits are needed to codify the most complex CU structure, and 320 bits to describe the same structure using the longest PU codes. This structure would be difficult to find in a real case because it involves the smallest CU partition size and two asymmetrical PU shapes.

Even in the worst case, where 320 bits are embedded, using an insertion rate of one bit per coefficient, in a $64 \times 64$ block with 4,096 transform coefficients, less than $10 \%$ of them will be needed for embedding.

\subsection{Multilevel Embedding Scheme}

Information is embedded into the DCT transform coefficients. Those coefficients belong to the block residuals generated during the motion compensation process. Thanks to the new structures introduced in HEVC, most of the transform coefficients are zero, this due to object shapes are closely approximated by using quad-tree structures and asymmetrical PUs; also, the transform process is carried out more efficiently by the newly introduced TUs. These zeros represent an excellent environment to implement a reversible embedding scheme.

The proposed method includes a multi-level embedding scheme where each LCU is evaluated previous to its modification, to decide if the information will be embedded into the first or second level, with the latter having more embedding space than the former but also introducing a higher distortion; if even the second level is not enough to embed the information, the embedding process is omitted. The EC method is prepared to perform concealment even when no information is available for the lost block. This embedding scheme is implemented to distribute the synthetic coefficients (created by embedding information into 
zero-coefficients) in a better way, and to avoid embedding the information only in the first TUs of the LCU. The number and amount of embedded information per level, is adjustable according to the application distortion constraints; in this work a two-level embedding scheme is used.

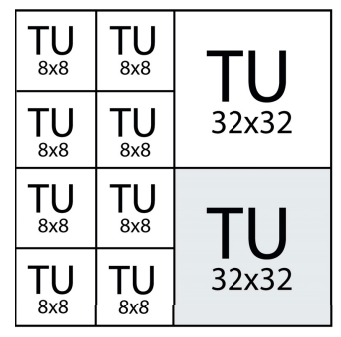

(a)

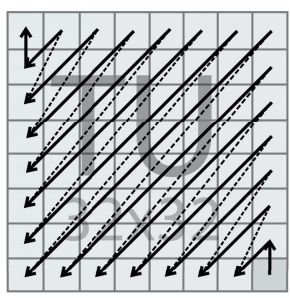

(b)

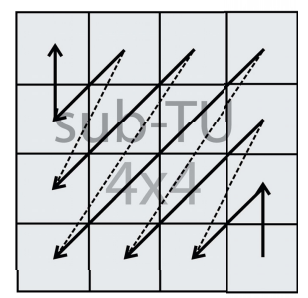

(c)

Fig. 4. Embedding scheme order. (a) CU partitioned into TUs for coding. (b) Each TU is divided into $4 \times 4$ blocks to perform embedding. The inverse diagonal order is used also at block level. (c) Embedding is performed in a inverse diagonal order at coefficient level.

Multilevel embedding scheme is described in figure 4, $4 \times 4$ transform coefficient blocks are used as embedding space in an inverse diagonal order (figure 4(c)). Exclusively zero-coefficients are used for embedding, and the last zerocoefficient of each block is used as flag to indicate if the block contains any information to be retrieved. A number of $4 \times 4$ blocks is assigned to each level according to the transform unit size (except by the $4 \times 4 \mathrm{TU}$ size), this is directly related to the amount of embedded information allowed per level. Table 1 reflects the number of blocks per level and transform sizes used in this work.

Table 1. Two-level embedding scheme configuration

\begin{tabular}{|c|c|c|}
\hline TU Size & Level 1 & Level 2 \\
\hline $4 \times 4$ & 4 coefficients & 4 coefficients \\
$8 \times 8$ & 1 block & 3 blocks \\
$16 \times 16$ & 4 blocks & 12 blocks \\
$32 \times 32$ & 16 blocks & 48 blocks \\
\hline
\end{tabular}

The embedding scheme aims at introducing only a small distortion by embedding the information in the last $4 \times 4$ block coefficients of each TU. Those coefficients represent high frequency information, the human visual system is less sensitive to high frequency distortions than to those in low frequency [8], so, most of the introduced distortion is not visible when the sequence is decoded using a normal decoder without the proposed DHEC feature. 


\subsection{Embedding Algorithm}

To achieve reversibility two rules are followed, first, only zero-coefficients are modified, and second, all the non-zero coefficient values are incremented by one. Over the zero-coefficients, even-odd signaling is used to embed the bit value. The embedding procedure is described by the flow chart in figure 5(a), the algorithm is applied starting from the last coefficients in each TU block, following the aforementioned inverse diagonal order.

There is no need to verify for overflow in the coefficient values, this is, because the complete process is performed after the quantization procedure. If lossless compression mode is used, it is necessary to add a verification step to avoid increasing the maximum coefficient values.

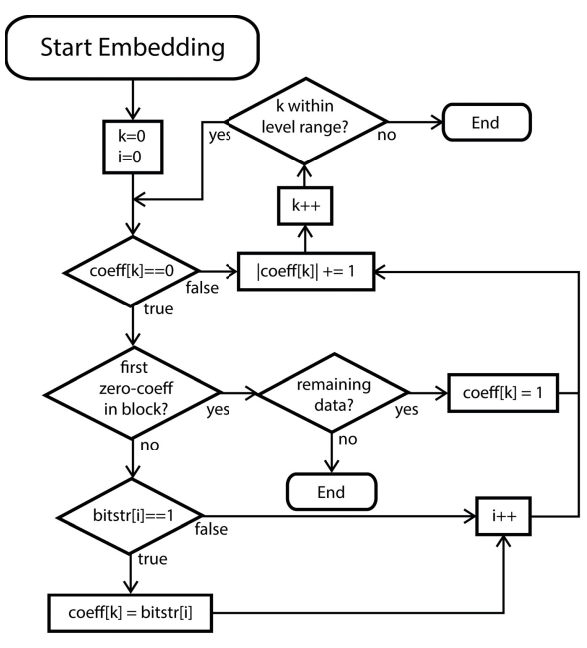

(a)

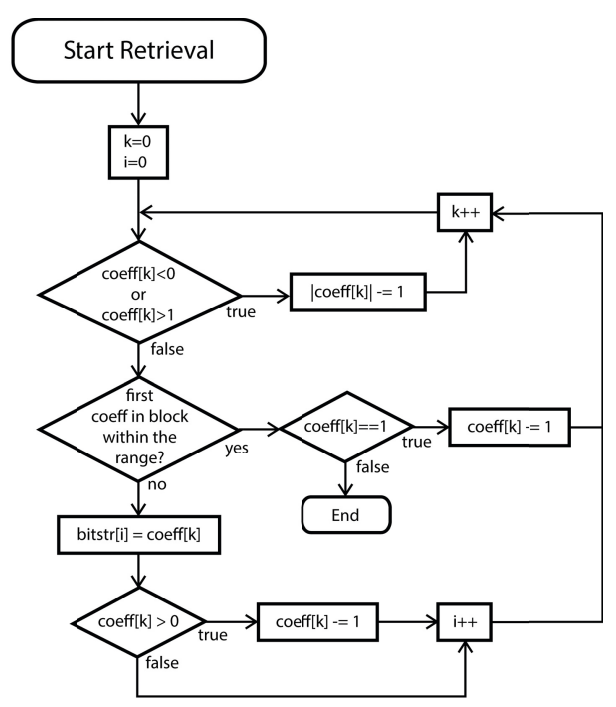

(b)

Fig. 5. Embedding and retrieval process flow charts; coef $f[k]$ and bitstr $[i]$ refer to the current coefficient and bit-string element, respectively. (a) Embedding process applied at TU level. (b) Retrieval process shown at TU level.

The embedding is carried out using an inverse diagonal order, during this process, each transform unit is partitioned into $4 \times 4$ blocks; the first zerocoefficient of each $4 \times 4$ block (in embedding order) is used as flag to signal if information was or not embedded, this coefficient might not be the first ( last in HEVC coding order and first in embedding order) to be found but the first zero-coefficient. As an example, the embedding order at block level into a $32 \times 32$ TU is shown in figure 4(b) and the order at coefficient level is shown in figure 4(c). 
In the proposed method, any complex slice interleaving scheme is avoided; those schemes interfere with the parallel encoding tools available in HEVC. Instead, the information belonging to a LCU is embedded within the LCU in the same position into the next slice, making it compatible with the wavefront parallel processing (WPP) feature in HEVC [5].

\section{Proposed Method: Decoder}

As it was mentioned in section 2.2, in some cases no information is embedded. The proposed scheme assigns a generic structure to the lost blocks whose concealment information is also lost or was not embedded due to lack of embedding space. The information retrieval process in the proposed method is integrated in the decoding stage; the information of each block is retrieved previously to the dequantization process, in this way, the host block is decoded without added distortions. This characteristic helps to make the embedding technique fully reversible, being capable of recovering the original block generated at encoder side, avoiding impairments between the decoded frame and the reference frame used during encoding. Obviously, this happens only when no errors occurred during transmission.

The original LCU structure, including its PUs is recovered from the retrieved information and assigned to the lost block; then, an outer boundary matching algorithm (OBMA), based on the mean of absolute differences (MAD) measure, is used to evaluate and choose the best replacement from a defined set of motion candidates, this process is carried out at PU level.

\subsection{Multilevel Retrieval Scheme}

The information retrieval process is simple and relies on flags that indicate if the $4 \times 4$ transform coefficient blocks contain or not embedded information. The retrieval process is described by the flow chart in figure 5(b); notice how the process is a mirrored version of the embedding algorithm, but without taking into account the levels it is fully guided by the block flags.

\subsection{Selecting the Best Neighbor}

The process of evaluating the best candidate is independent from the block structure origin, the structure can be the one generated from the retrieved information, or a generic one assigned to all the lost blocks whose information was lost or not embedded. The best neighbor selection has two main characteristics: the candidate list and the MAD calculation. These two characteristics are explained next:

- Candidate list. The candidate list includes the top, top-left, left, bottom-left, bottom, right and top-right neighboring PUs within the same frame. It also includes zero-motion and collocated neighbors in both the next and previous 
frames. Anyway, not all candidates are evaluated; a maximum of 4 neighbors, plus the zero-motion case, are evaluated per PU partition. Also, each PU partition has its own candidates depending on its shape and position. The PU partition shapes, position, and their possible neighboring candidates are shown in figure 6, all the presented shapes can use the collocated and zeromotion candidates.

In figure 6, each partition has an arrow, this arrow signals the called most important neighbor (MIN); this means, that the partition is more likely to share motion information with that specific partition than with any other. It is common to some neighboring partitions not to be available, this is due to the concealment order (up-down, left-right). To improve concealment, a reach-forward scheme is implemented; this means that if the neighboring PU is not available, and it is the case of a MIN, the next PU along the same direction is reached and taken as a candidate. This technique is not always useful and its use is mostly restricted to cases when only one LCU is coded per slice.

- OBMA based on MAD. The MAD between the outer pixels of each PU partition and the outer pixels of each candidate is used to select the best replacement. The pixels involved in the calculation of MAD depend on the partition shape and position; in figure 7, each partition and the pixels involved in the MAD calculation are signaled. This selection is based on the way the partitioning scheme works in HEVC; if two partitions within the same CU share the same motion information, there is no reason of the partition to exist, a bigger partition would had been coded instead. Therefore, PU partitions withing the same $\mathrm{CU}$ are ignored in the motion candidate list creation and in the MAD calculation.
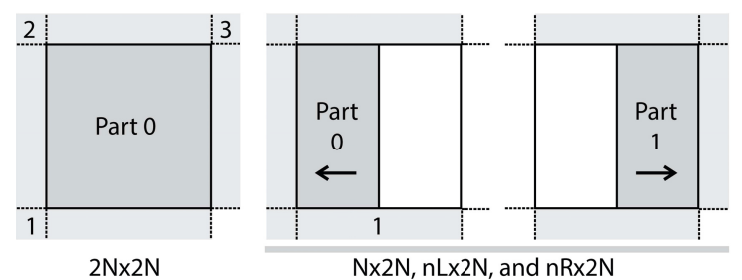

$\mathrm{N} \times 2 \mathrm{~N}, \mathrm{~nL} \times 2 \mathrm{~N}$, and $\mathrm{nR} \times 2 \mathrm{~N}$

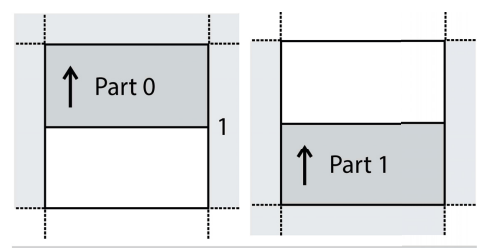

$2 \mathrm{NxN}, 2 \mathrm{NxnU}$, and $2 \mathrm{NxnD}$

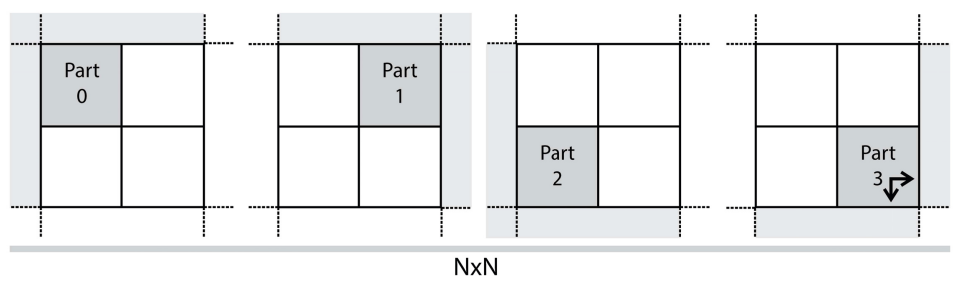

Fig. 6. Different PUs and their candidates. The partition number and its neighboring motion candidates are shown in a darker color, arrows signal the direction over which the reach-forward technique is used. 


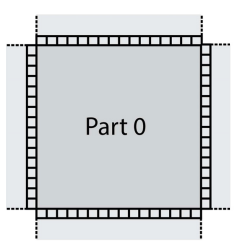

$2 \mathrm{~N} \times 2 \mathrm{~N}$

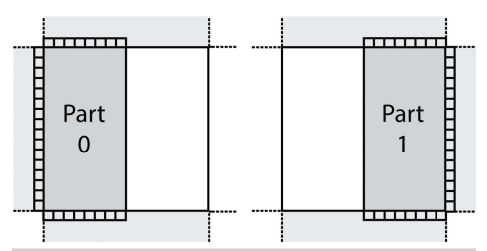

$\mathrm{N} \times 2 \mathrm{~N}, \mathrm{~nL} \times 2 \mathrm{~N}$, and $\mathrm{nR} \times 2 \mathrm{~N}$

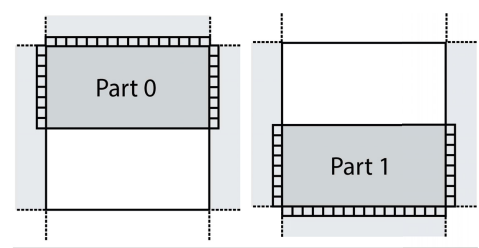

$2 \mathrm{NxN}, 2 \mathrm{NxnU}$, and $2 \mathrm{NxnD}$

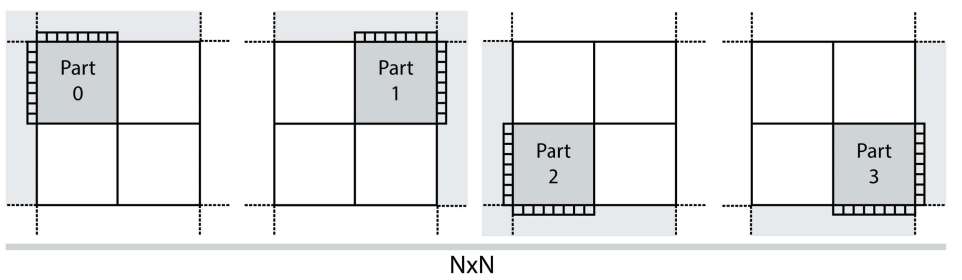

Fig. 7. Different PU shapes and the pixels involved in the calculation of the MAD measure, used to select the best replacement for the lost part

\section{Experiments and Results}

Achieving a fair comparison between the proposed DHEC method and the state of the art approaches is not an easy task. Due to the new features introduced in HEVC and those that were removed in comparison with H.264/AVC, the direct implementation of most of the previous methods is not possible. A widely known temporal error concealment (TEC) method was proposed by Wang et al. 9], it is based on candidate evaluation using boundary matching algorithm (BMA) [14]. The concealment results achieved with [9] are fair, so, it was adopted as the non-normative TEC method in the H.264/AVC joint model (JM); many different works use BMA [14] or a simpler version for comparison [15 20], making of it a good reference for new works.

In this section a comparison between the concealment results of the proposed DHEC method and [9] is presented; this provides a reference point to evaluate the performance of the proposed method. All the experiments were carried out over four different video sequences: Traffic, Kimono, KristenAndSara, and RaceHorses; they were chosen based on their resolution and motion characteristics. The four test sequences were exposed to different random damage levels, i.e. packet losses were simulated using different random patterns, during this process only inter coded frames were affected; all losses were at slice level (simulating one slice per packet in a scenario were retransmission is not feasible), having one LCU per slice. All the experiments were performed using the HEVC's TMuC in its HM9.1rc1 version [11], also, all videos were coded using the recommendations given in [10], using the Low-delay B - Main configuration, and disabling the Sample Adaptive Offset (SAO) and Sign Hiding features. 
Table 2. Error concealment results with a $1 \%$ of random loss; values in bold represent the best result for each experiment. The PSNR [2] and the SSIM [1] metrics are used to evaluate and compare the quality of the concealed sequences

\begin{tabular}{cccccccc}
\hline Sequence & Resolution HM9.1rc1 & - Zero & Wang, et al. [9] & \multicolumn{3}{c}{ Proposed DHEC } \\
\hline & & PSNR (dB) & SSIM & PSNR $(\mathrm{dB})$ & SSIM & PSNR (dB) & SSIM \\
Traffic & $2560 \times 1600$ & 22.2249 & 0.8677 & 29.8122 & 0.9639 & $\mathbf{3 3 . 2 0 3 1}$ & $\mathbf{0 . 9 8 4 1}$ \\
Kimono & $1920 \times 1080$ & 24.0473 & 0.8861 & 32.5780 & 0.9543 & $\mathbf{3 4 . 1 7 8 9}$ & $\mathbf{0 . 9 6 6 9}$ \\
KristenAndSara & $1280 \times 720$ & 16.2563 & 0.6866 & 34.5961 & 0.9755 & $\mathbf{3 5 . 3 1 6 1}$ & $\mathbf{0 . 9 8 0 0}$ \\
RaceHorses & $832 \times 480$ & 20.0839 & 0.7855 & 26.4078 & 0.8825 & $\mathbf{2 8 . 7 9 0 2}$ & $\mathbf{0 . 9 2 1 7}$ \\
\hline
\end{tabular}

Table 3. Error concealment results with $5 \%$ of random loss; values in bold represent the best result for each experiment. The PSNR [2] and the SSIM [1] metrics are used to evaluate and compare the quality of the concealed sequences

\begin{tabular}{cccccccc}
\hline Sequence & Resolution HM9.1rc1 & Zero & Wang, et al. [9] & \multicolumn{2}{c}{ Proposed DHEC } \\
\hline & & PSNR (dB) & SSIM & PSNR $(\mathrm{dB})$ & SSIM & PSNR (dB) & SSIM \\
Traffic & $2560 \times 1600$ & 16.2081 & 0.5772 & 24.5597 & 0.8819 & $\mathbf{2 7 . 5 6 8 0}$ & $\mathbf{0 . 9 3 5 4}$ \\
Kimono & $1920 \times 1080$ & 18.1355 & 0.6355 & 27.3605 & 0.8363 & $\mathbf{2 8 . 7 5 0 8}$ & $\mathbf{0 . 8 7 8 2}$ \\
KristenAndSara & $1280 \times 720$ & 11.2851 & 0.4034 & 29.7259 & 0.9287 & $\mathbf{2 9 . 8 4 8 7}$ & $\mathbf{0 . 9 3 8 5}$ \\
RaceHorses & $832 \times 480$ & 14.5185 & 0.4709 & 21.4956 & 0.6879 & $\mathbf{2 3 . 5 3 7 4}$ & $\mathbf{0 . 7 6 6 0}$ \\
\hline
\end{tabular}

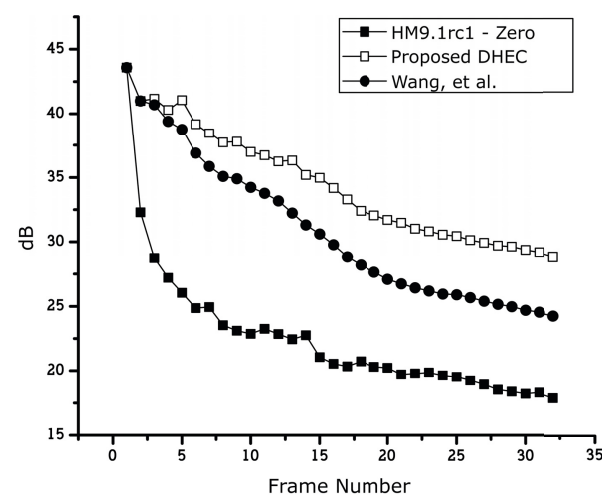

Fig. 8. Error propagation through frames due to inter-prediction coding with $1 \%$ of damage. Low quality concealment in a current frame introduces distortions into following frames due to inter prediction, this effect heavily degrades the sequence quality

Table 2 shows the comparison between the results of the proposed method, the method proposed by Wang et al. [9], and zero substitution (lost blocks are substituted by black areas). It can be seen that the proposed method performs better in the sequences Traffic and RaceHorses, both classified as high motion, with a maximum PSNR gain of $3.39 \mathrm{~dB}$ over [9]; it is noticeable that the same result pattern is maintained in the SSIM measurements. In table 3 the proposed method achieves better concealment results in the same two sequences. Nonetheless, it has to be mentioned that the proposed method introduced an average 
increment of $3.83 \%$ in the bit rate of the sequences; the increment has a strong relation with the video motion characteristics and image detail.

One of the main reasons to perform concealment over damaged frames is to avoid the error propagation due to inter and intra prediction. In figure 8, the error propagation through frames is shown, notice how the proposed method helps to control the propagation better than Wang et al. [9].

\section{Conclusions}

New features were included in the new HEVC standard; these features are not compatible with most of the state of the art video error concealment methods. In this work we presented a data hiding based error concealment approach; with the aid of information available during encoding stage such as the LCU structure, and performing the concealment at PU level, it is possible to obtain superior results to [9], especially in high motion videos. The presented method was specially designed to be compatible with the new features in $\mathrm{HEVC}$, and can be used to provide error concealment to the new generation of video applications.

Acknowledgments. The authors would like to thank to CONACYT for the grant number 261243 .

\section{References}

1. Wang, Z., Bovik, A.C., Sheikh, H.R., Simoncelli, E.P.: Image quality assessment: from error visibility to structural similarity. IEEE Transactions on Image Processing 13(4), 600-612 (2004)

2. ITU-T.: J.247: Objective perceptual multimedia video quality measurement in the presence of a full reference (2008) (online) http://www.itu.int/

3. New video codec to ease pressure on global networks - press release, http://www.itu.int/net/pressoffice/press/_releases/2013/01.aspx

4. Pourazad, M., Doutre, C., Azimi, M., Nasiopoulos, P.: HEVC: The New Gold Standard for Video Compression: How Does HEVC Compare with H.264/AVC? IEEE Consumer Electronics Magazine 1(3), 36-46 (2012)

5. Sullivan, G.J., Ohm, J., Han, W., Wiegand, T.: Overview of the High Efficiency Video Coding (HEVC) Standard. IEEE Transactions on Circuits and Systems for Video Technology 22(12), 1649-1668 (2012)

6. Sole, J., Joshi, R., Nguyen, N., Ji, T., et al.: Transform Coefficient Coding in HEVC. IEEE Transactions on Circuits and Systems for Video Technology 22(12), 1765-1777 (2012)

7. Kim, I.-K., McCann, K., Sugimoto, K., Bross, B., Han, W.: High Efficiency Video Coding (HEVC) Test Model 9 Encoder Description. Document of Joint Collaborative Team on Video Coding, JCTVC-K1002v2 (2012)

8. Richardson, I.E.G.: Video Codec Design, Developing Image and Video Compression System. John Wiley \& Sons Ltd., England (2002)

9. Wang, Y.-K., Hannuksela, M.M., Varsa, V., Hourunranta, A., Gabbouj, M.: The error concealment feature in the H.26L test model. In: International Conference on Image Processing, vol. 2, pp. II-729-II-732. IEEE (2002) 
10. Bossen, F.: Common test conditions and software reference configurations. Document of Joint Collaborative Team on Video Coding, JCTVC-K1100 (2012)

11. HEVC's Test Model under Consideration, Software Repository, https://hevc.hhi.fraunhofer.de/svn/svn_HEVCSoftware/tags/HM-9.1rc1/

12. Wiegand, T., Sullivan, G.J., Bjontegaard, G., Luthra, A.: Overview of the H.264/AVC video coding standard. IEEE Transactions on Circuits and Systems for Video Technology 13(7), 560-576 (2003)

13. Pyun, J.-Y., Lee, J.-S., Jeong, J.-W., Jeong, J.-H., Ko, S.-J.: Robust error concealment for visual communications in burst-packet-loss networks. IEEE Transactions on Consumer Electronics 49(4), 1013-1019 (2003)

14. Lam, W.-M., Reibman, A.R., Liu, B.: Recovery of lost or erroneously received motion vectors. In: IEEE International Conference on Acoustics, Speech, and Signal Processing, vol. 5, pp. 417-420. IEEE (1993)

15. Seth, K., Kamakoti, V., Srinivasan, S.: Efficient Motion Vector Recovery Algorithm for H.264 Using B-Spline Approximation. IEEE Transactions on Broadcasting 56(4), 467-480 (2010)

16. Kim, D., Yang, S., Jeong, J.: A new temporal error concealment method for H.264 using adaptive block sizes. In: IEEE International Conference on Image Processing, vol. 3, pp. III-928-III-931. IEEE (2005)

17. Xu, Y., Zhou, Y.: H.264 video communication based refined error concealment schemes. IEEE Transactions on Consumer Electronics 50(4), 1135-1141 (2004)

18. Zheng, J., Chau, L.-P.: Efficient motion vector recovery algorithm for H.264 based on a polynomial model. IEEE Transactions on Multimedia 7(3), 507-513 (2005)

19. Zhou, J., Yan, B., Gharavi, H.: Efficient Motion Vector Interpolation for Error Concealment of H.264/AVC. IEEE Transactions on Broadcasting 57(1), 75-80 (2011)

20. Chen, T., Zhang, X., Shi, Y.-Q.: Error concealment using refined boundary matching algorithm. In: International Conference on Information Technology: Research and Education, pp. 55-59. IEEE (2003) 